\title{
Diferencias en las prevalencias de desnutrición al usarse referencias nacionales e internacionales
}

\author{
Evelia Edith Oyhenart ${ }^{1,2}$ y Alicia Bibiana Orden ${ }^{2}$
}

Forma de citar Oyhenart EE, Orden AB. Diferencias en las prevalencias de desnutrición al usarse referencias nacionales e internacionales. Rev Panam Salud Publica. 2005;18(3):157-62.

RESUMEN Objetivo. Evaluar si el empleo de dos referencias distintas (internacional y nacional) ocasiona diferencias en las prevalencias de emaciación y retraso del crecimiento en una muestra de niños de 8 a 14 años.

Métodos. Se realizó un estudio transversal en una muestra de 1470 escolares de ambos sexos de la ciudad de La Plata (Provincia de Buenos Aires, Argentina). A partir de los datos de peso y talla se calcularon las puntuaciones $Z$ respecto a los valores de referencia de la primera y la segunda encuesta estadounidense de salud y nutrición (National Health and Nutrition Examination Survey, NHANES I y II), y de la Sociedad Argentina de Pediatría. Las prevalencias de emaciación y retraso del crecimiento se calcularon tomando un punto de corte de dos desviaciones estándar $(D E)$ por debajo de la mediana de referencia. Se compararon las prevalencias mediante la prueba de ji al cuadrado con corrección de Yates y se calcularon la razón de posibilidades (odds ratio, RP) y los intervalos de confianza del 95\% (IC95\%).

Resultados. La referencia internacional se asoció positivamente a una mayor prevalencia de retraso del crecimiento, tanto en la muestra total $(R P=5,12 ; I C 95 \%: 3,27$ a 8,05) como en varones $(R P=4,36$; IC95\%: 2,43 a 7,93$)$ y en mujeres ( $R P=6,32 ; I C 95 \%: 3,10$ a 13,27). La prevalencia de emaciación fue similar con ambas referencias. La distribución de la talla para la edad presentó un sesgo a la izquierda respecto de la referencia internacional y a la derecha respecto de la nacional, mientras que la del peso para la talla se superpuso a ambas referencias.

Conclusiones. Las diferencias en las prevalencias de retraso del crecimiento calculadas según las dos referencias utilizadas reflejan cuadros disimiles del estado nutricional de la población evaluada. El incremento de la talla de los niños respecto a la referencia nacional apunta a la presencia de una tendencia secular positiva y puede explicar parte de las diferencias en la prevalencia de retraso del crecimiento.

Palabras clave Estándares de referencia, estado nutricional, desnutrición.

Las mediciones antropométricas son instrumentos útiles para evaluar el estado nutricional individual y pobla- cional. El uso de una referencia posibilita comparar el crecimiento y el estado nutricional a diferentes edades.

\footnotetext{
1 Facultad de Ciencias Naturales y Museo, Universidad Nacional de La Plata (UNLP), La Plata, Provincia de Buenos Aires, Argentina. La correspondencia debe dirigirse a: Evelia Edith Oyhenart, CIGEBA, Facultad de Ciencias Veterinarias, Universidad Nacional de la Plata, Calles 60 y 118,
}

CC296, (B1094AAO), La Plata, Provincia de Buenos Aires, Argentina.

2 Facultad de Ciencias Veterinarias, Universidad Nacional de la Plata, La Plata, Provincia de Buenos Aires, y Consejo Nacional de Investigaciones Científicas y Técnicas (CONICET), Argentina.
Sin embargo, existen numerosos interrogantes concernientes al enfoque, el análisis y la interpretación de las medidas antropométricas en la evaluación del estado nutricional. Hasta ahora, no hay consenso con respecto al hecho de que el empleo de una única referencia internacional sea suficiente para evaluar el crecimiento de todos 
los niños, prescindiendo de los factores genéticos (1). Así, mientras Waterlow et al. (2) recomendaron utilizar los datos del National Center for Health Statistics (Centro Nacional de Estadísticas Sanitarias, NCHS) de los Estados Unidos de América como estándar de referencia internacional, otros investigadores señalaron que los valores de referencia provenientes de la propia población son más adecuados (3-6). Johnston y Ouyang (7) presentaron los argumentos a favor de una $u$ otra posición. Por una parte, estos autores sostienen que los estándares de referencia internacionales, como el del NCHS, construidos en países desarrollados, conducen a sobrestimar la prevalencia de desnutrición cuando se emplean en países en desarrollo. Por otra, señalan que el empleo de referencias locales no es totalmente preciso, dada la alta proporción de personas desnutridas que puede llegar a integrar la muestra. Otro factor que debe considerarse es la posibilidad de que exista una tendencia secular del creci- miento en un período relativamente breve, lo que hace que la referencia local sea menos apta para la identificación de trastornos nutricionales y del crecimiento $(8,9)$.

En nuestro país, la Sociedad Argentina de Pediatría y el Ministerio de Salud acordaron utilizar para la evaluación del crecimiento y el estado nutricional una serie de curvas, cuyos datos se publicaron en 1987 (10). Para construir estas curvas se emplearon datos provenientes de estudios longitudinales y transversales realizados en dos ciudades, algunos de los cuales datan de hace más de 35 años (11-14). En un trabajo reciente, del Pino et al. (15) mantuvieron la recomendación de utilizar dichas referencias en forma confiable para la franja etárea comprendida entre los $0 \mathrm{y}$ 5 años, pero advirtieron de la necesidad de realizar estudios a edades más avanzadas para comprobar la existencia de una eventual tendencia secular.

El objetivo del presente estudio es evaluar si el empleo de dos referencias distintas — una internacional y la otra nacional- ocasiona diferencias en las prevalencias de emaciación (peso bajo para la talla) y retraso del crecimiento (talla baja para la edad) en una muestra de niños de 8 a 14 años de edad.

\section{MATERIALES Y MÉTODOS}

Se realizó un estudio transversal con una muestra representativa de la población escolar matriculada en el ciclo de educación general básica de la ciudad de La Plata (Provincia de Buenos Aires, Argentina). El tamaño muestral se determinó ajustado por población finita, con un nivel de confianza del $95 \%$ y un error de muestreo del $2 \%$. La muestra se obtuvo de forma aleatoria y se estratificó por edad y sexo. Se estudió a 1470 niños sanos de ambos sexos (740 varones y 730 mujeres) entre los 8 y 14 años de edad (cuadro 1). En todos los casos se obtuvo el consentimiento válido de los padres o los tutores.

El estado nutricional de los escolares se determinó a partir de dos indicadores: talla para la edad y peso para la

CUADRO 1. Comparación de las prevalencias totales y por sexo y edad de retraso del crecimiento y de emaciación, calculadas según las referencias nacional e internacional

\begin{tabular}{|c|c|c|c|c|c|c|c|c|c|c|c|c|c|}
\hline & \multirow[b]{3}{*}{$n$} & \multicolumn{6}{|c|}{ Talla baja para la edad (retraso del crecimiento) } & \multicolumn{6}{|c|}{ Peso bajo para la talla (emaciación) } \\
\hline & & \multicolumn{2}{|c|}{ Prevalencia (\%) } & \multirow[b]{2}{*}{$\chi^{2}$} & \multirow[b]{2}{*}{$P$} & \multirow[b]{2}{*}{$\mathrm{RP}^{\mathrm{a}}$} & \multirow[b]{2}{*}{ IC95\%b } & \multicolumn{2}{|c|}{ Prevalencia (\%) } & \multirow[b]{2}{*}{$\chi^{2}$} & \multirow[b]{2}{*}{$P$} & \multirow[b]{2}{*}{$\mathrm{RP}$} & \multirow[b]{2}{*}{ IC95\% } \\
\hline & & $\begin{array}{c}\text { Referencia } \\
\text { internacional }\end{array}$ & $\begin{array}{c}\text { Referencia } \\
\text { nacional }\end{array}$ & & & & & $\begin{array}{c}\text { Referencia } \\
\text { internacional }\end{array}$ & $\begin{array}{c}\text { Referencia } \\
\text { nacional }\end{array}$ & & & & \\
\hline Muestra total & 1470 & 8,4 & 1,8 & 67,47 & 0,0000 & 5,12 & $3,27-8,05$ & 0,5 & 1,1 & 3,30 & 0,0694 & 0,41 & $0,15-1,06$ \\
\hline \multicolumn{14}{|l|}{ Varones } \\
\hline Total & 730 & 8,9 & 2,2 & 31,38 & 0,0000 & 4,36 & $2,43-7,93$ & 0,5 & 1,2 & 3,37 & 0,0662 & 0,22 & $0,03-1,08$ \\
\hline 8 & 87 & 8,0 & 1,1 & 3,28 & 0,0703 & 7,53 & $0,90-166,34$ & 0,0 & 1,1 & $N A^{c}$ & & & \\
\hline 9 & 141 & 22,7 & 6,4 & 15,10 & 0,0001 & 4,31 & $1,87-10,18$ & 0,7 & 0,7 & 0,50 & 0,4779 & 1,00 & $0,00-36,95$ \\
\hline 10 & 120 & 4,2 & 2,5 & 0,13 & 0,7192 & 1,70 & $0,34-9,19$ & 0,0 & 0,8 & NA & & & \\
\hline 11 & 121 & 1,7 & 0,8 & 0,00 & 1.0000 & 2,02 & $1,14-56,95$ & 0,8 & 3,3 & 0,82 & 0,3661 & 0,24 & $0,01-2,36$ \\
\hline 12 & 97 & 4,3 & 1,0 & 2,62 & 0,1056 & 4,17 & $0,81-28,82$ & 0,0 & 0,0 & NA & & & \\
\hline 13 & 80 & 6,3 & 1,3 & 1,56 & 0,2119 & 5,27 & $0,58-121,96$ & 0,0 & 1,3 & NA & & & \\
\hline 14 & 84 & 11,9 & 1,2 & 6,23 & 0,0125 & 11,22 & $1,42-239,73$ & 0,0 & 1,3 & NA & & & \\
\hline \multicolumn{14}{|l|}{ Mujeres } \\
\hline Total & 740 & 8,0 & 1,4 & 36,50 & 0,0000 & 6,32 & $3,10-13,27$ & 1,7 & 1,4 & 1,00 & 0,3179 & 0,61 & $0,21-1,75$ \\
\hline 8 & 100 & 7,1 & 0,0 & 8,00 & 0,0047 & 7,53 & $1,61-48,65$ & 2,0 & 2,0 & 0,26 & 0,6135 & 1,00 & $0,10-10,17$ \\
\hline 9 & 111 & 4,5 & 0,9 & 1,54 & 0,2144 & 5,19 & $0,58-119,34$ & 0,0 & 1,8 & NA & & & \\
\hline 10 & 116 & 5,2 & 1,7 & 1,17 & 0,2804 & 3,11 & $0,55-22,79$ & 1,7 & 3,4 & 0,17 & 0,6791 & 0,49 & $0,06-3,20$ \\
\hline 11 & 120 & 5,8 & 0,0 & 7,92 & 0,0049 & 7,43 & 1,59-47,88 & 0,0 & 0,0 & NA & & & \\
\hline 12 & 103 & 7,7 & 0,0 & 9,94 & 0,0016 & 8,67 & $1,88-55,29$ & 1,0 & 2,1 & 0,00 & 0,9515 & 0,46 & $0,02-6,62$ \\
\hline 13 & 94 & 19,1 & 4,3 & 8,70 & 0,0032 & 5,33 & $1,60-19,53$ & 0,0 & 0,0 & NA & & & \\
\hline 14 & 96 & 8,3 & 3,1 & 1,54 & 0,2142 & 2,82 & $0,65-13,89$ & 0,0 & 0,0 & NA & & & \\
\hline
\end{tabular}

RP: razón de posibilidades.

b IC95\%: intervalo de confianza del 95\%.

c NA: no se aplica. 
talla. La edad de cada niño se obtuvo del documento nacional de identidad. El peso corporal se midió en kilogramos con una balanza digital portátil (10 g de precisión) que se calibró al inicio de cada sesión. En todos los casos los niños vistieron ropa liviana cuyo peso se descontó del peso total. La talla se midió en centímetros utilizando un antropómetro vertical (1 $\mathrm{mm}$ de precisión), con el niño descalzo y orientando la cabeza en el plano de Frankfort.

Todas las mediciones se realizaron en horario escolar, durante los meses de abril y noviembre de 2003, por uno de los autores previamente entrenado, según protocolos estandarizados (16). Para determinar el error intraobservador cada medición se realizó dos veces. La concordancia entre ambas mediciones se evaluó mediante el coeficiente de correlación intraclase (CCI). Se consideraron aceptables los valores de CCI superiores a 0,75 (17).

Los valores de peso y talla se transformaron en puntuaciones $Z$ respecto a los datos de la primera y la segunda encuesta estadounidense de salud y nutrición (National Health and Nutrition Examination Survey, NHANES I y II), como referencia internacional (18), y de la Sociedad Argentina de Pediatría (10), como referencia nacional. Las prevalencias de emaciación y retraso del crecimiento se calcularon tomando un punto de corte de dos desviaciones estándar (DE) por debajo de la mediana de referencia (19).

Las prevalencias obtenidas según las referencias internacional y nacional se compararon mediante la prueba de ji al cuadrado $\left(\chi^{2}\right)$ con corrección de Yates (nivel de significación: $P<0,05$ ). Dado que los valores $P$ no permiten conocer la magnitud ni la precisión del efecto observado (20), se calcularon la razón de posibilidades (odds ratio, RP) y los respectivos intervalos de confianza del 95\% (IC95\%). Los datos se procesaron mediante los programas estadísticos SPSS 7.0 y Epi-Info 6.04.

\section{RESULTADOS}

En el cuadro 1 se presentan las prevalencias totales y por sexo y edad de los indicadores estudiados. El análisis de la muestra total evidenció diferencias significativas $(P \leq 0,01)$ en la prevalencia de talla baja para la edad según la referencia empleada. Con la referencia internacional se obtuvo una mayor prevalencia de retraso del crecimiento, tanto en la muestra total ( $\mathrm{RP}=$ 5,12; IC95\%: 3,27 a 8,05) como en los varones ( $\mathrm{RP}=4,36$; IC95\%: 2,43 a 7,93 ) y las mujeres $(\mathrm{RP}=6,32$; IC95\%: 3,10 a 13,27$)$.

Al estratificar por edad también se observó que con el empleo de la referencia internacional era mayor la prevalencia de talla baja para la edad a los $9(P=0,0001 ; \mathrm{RP}=4,31$; IC95\%:1,87 a $10,18)$ y 14 años $(P=0,0125 ; R P=11,22$; IC95\%: 1,42 a 239,73) en los varones y a $\operatorname{los} 8(P=0,0047 ; \mathrm{RP}=7,53$; IC95\%: 1,61 a 48,65), $11(P=0,0049 ; \mathrm{RP}=7,43$; IC95\%: 1,59 a 47,88), $12(P=0,0016$; RP $=8,67$; IC95\%: 1,88 a 55,29) y 13 años $(P=0,0032 ; \mathrm{RP}=5,33$; IC95\%:1,60 a $19,53)$ en las mujeres (cuadro 1).

Las diferencias entre las prevalencias de bajo peso para la talla no fueron estadísticamente significativas $(P>$ $0,05)$ en ninguna de las comparaciones (cuadro 1).

En la figura 1 se representan las distribuciones, para cada sexo, de las puntuaciones $Z$ de los indicadores empleados. La distribución relativa al peso para la talla se superpuso con la de ambas referencias. En cambio, la distribución relativa a la talla para la edad presentó en ambos sexos un sesgo a la izquierda con respecto a la referencia internacional y a la derecha con respecto a la nacional.

\section{DISCUSIÓN}

Las prevalencias de emaciación determinadas según ambas referencias fueron menores que las proporciones de casos que sería esperable encontrar por debajo de 2 DE respecto a la mediana en una población de niños sanos y bien alimentados (2).

Basándose en la clasificación de Gorstein et al (19), las prevalencias de retraso del crecimiento determinadas según la referencia nacional resultaron muy bajas $(1,8 \%)$ y las correspondientes a la referencia internacional, moderadas $(8,4 \%)$. Estos resultados concuer- dan con los obtenidos en otras poblaciones latinoamericanas, caracterizadas por altas proporciones de retraso del crecimiento y bajas proporciones de emaciación (21).

La estratificación por sexo reflejó lo observado en la muestra total, en la cual la prevalencia de retraso del crecimiento fue mayor cuando se usó la referencia internacional. El análisis por edad, no obstante, evidenció una variación de la magnitud de las diferencias obtenidas al emplear las distintas referencias. Así, en los varones, la diferencia fue mayor a los 14 años $(\mathrm{RP}=$ $11,22)$ que a los 9 años $(R P=4,31)$, y en las mujeres, a los 12 años $(R P=8,67)$ que a $\operatorname{los} 8,11$ y 13 años $(R P=7,53$, 7,43 y 5,33 , respectivamente). Estos resultados concuerdan con los obtenidos por Eckhardt y Adair (22), quienes compararon los datos provenientes de una muestra de niños filipinos con los valores de referencia del NCHS de 1977 y de los Centros para el Control y la Prevención de Enfermedades (CDC) de 2000, y hallaron una variación en la magnitud y el signo de la puntuación $Z$ y de las prevalencias de retraso del crecimiento en las distintas edades.

Al analizar la distribución de las puntuaciones $Z$, se observan dos situaciones. Respecto al indicador peso para la talla, la distribución de la población en estudio se superpone a las de ambas referencias, lo que hace imposible diferenciar la población de origen. Respecto al indicador talla para la edad, la distribución de la población en estudio ocupa una posición intermedia, lo que dificulta la elección de la referencia más apropiada.

La elección de un estándar de referencia está sin duda condicionada por la existencia de cambios seculares. En consecuencia, si existió una tendencia secular en la población, cualquier serie de datos o valores de referencia resulta de poca utilidad para realizar un seguimiento del crecimiento. El problema se complica aun más si se tiene en cuenta que la tendencia secular no siempre es rectilínea ni necesariamente positiva (9). En numerosos países de América Latina se ha hallado un incremento de la talla media (2325). En Argentina, los escasos estudios 
FIGURA 1. Distribución de las puntuaciones $Z$ del peso para la talla y la talla para la edad respecto a las referencias internacional (curva continua) y nacional (curva discontinua)

Peso para la talla, varones

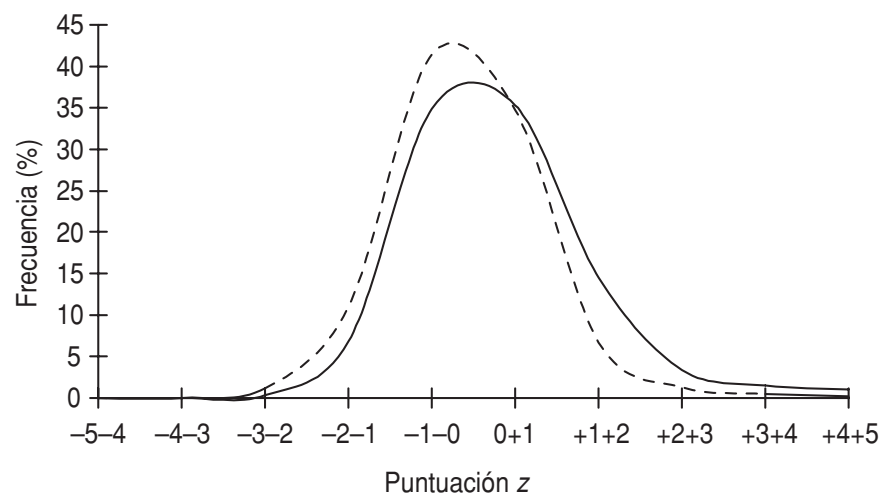

Talla para la edad, varones

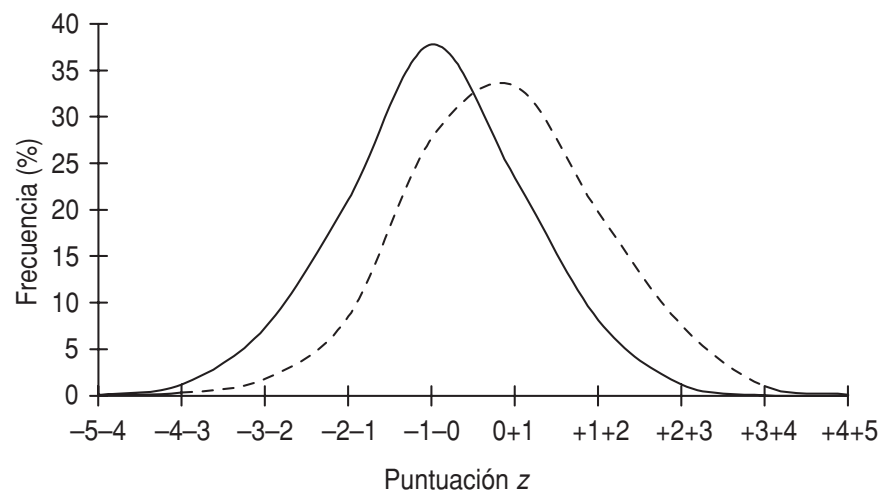

Peso para la talla, mujeres

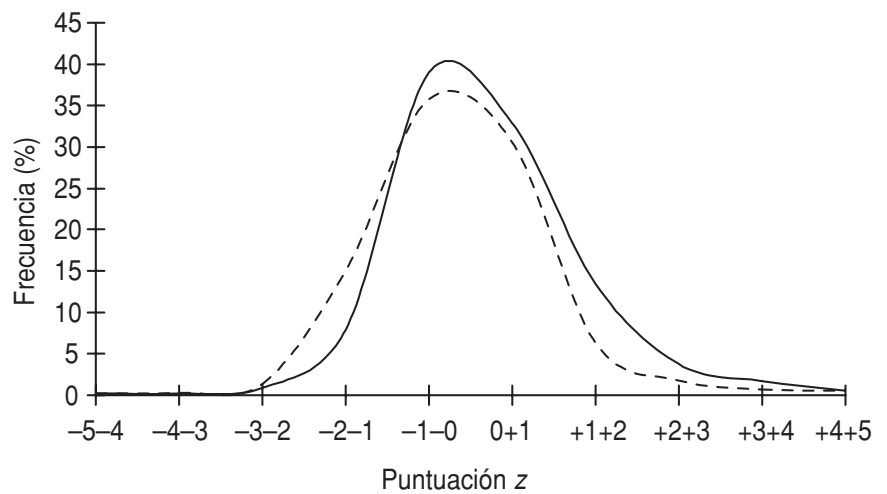

Talla para la edad, mujeres

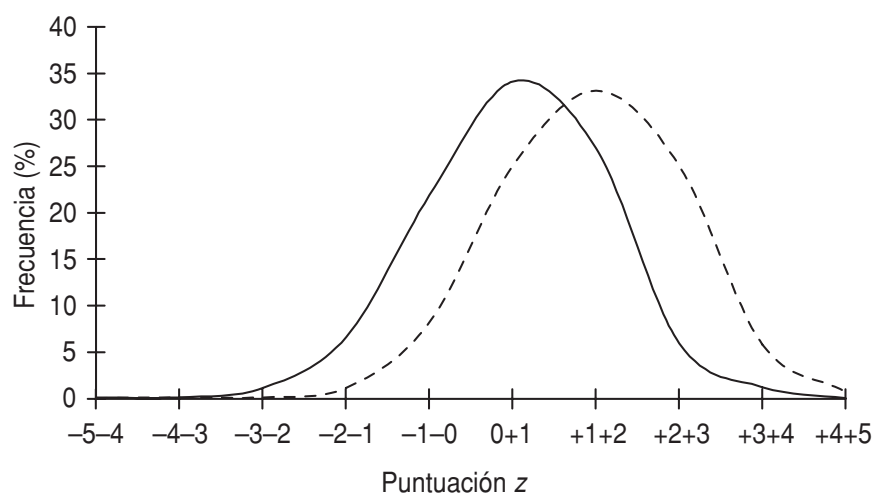

FIGURA 2. Talla de los niños de la muestra estudiada (círculos y línea continua) y de la referencia nacional (triángulos y línea discontinua). (Los valores se expresan como la mediana (percentil 50) \pm desviación estándar)

Varones

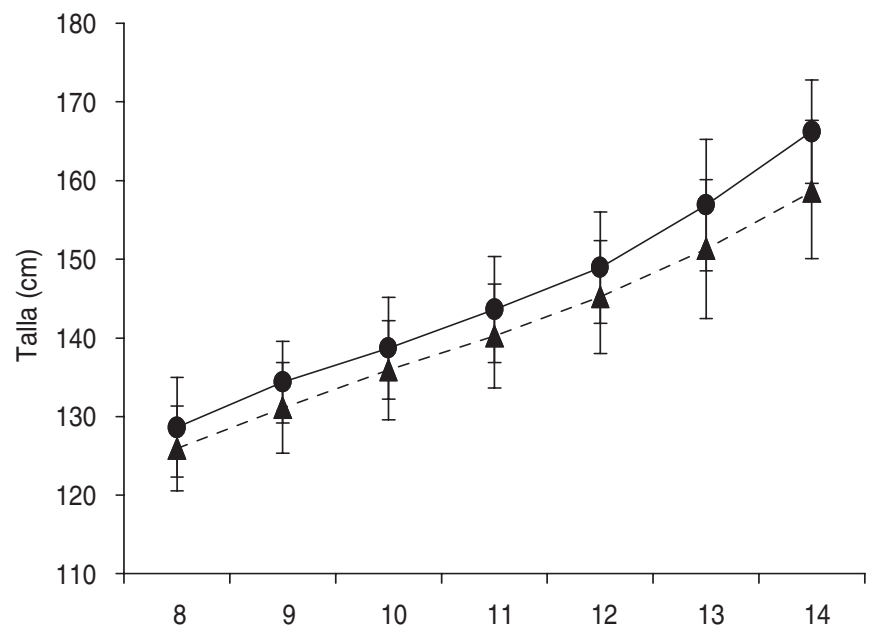

Edad, años
Mujeres

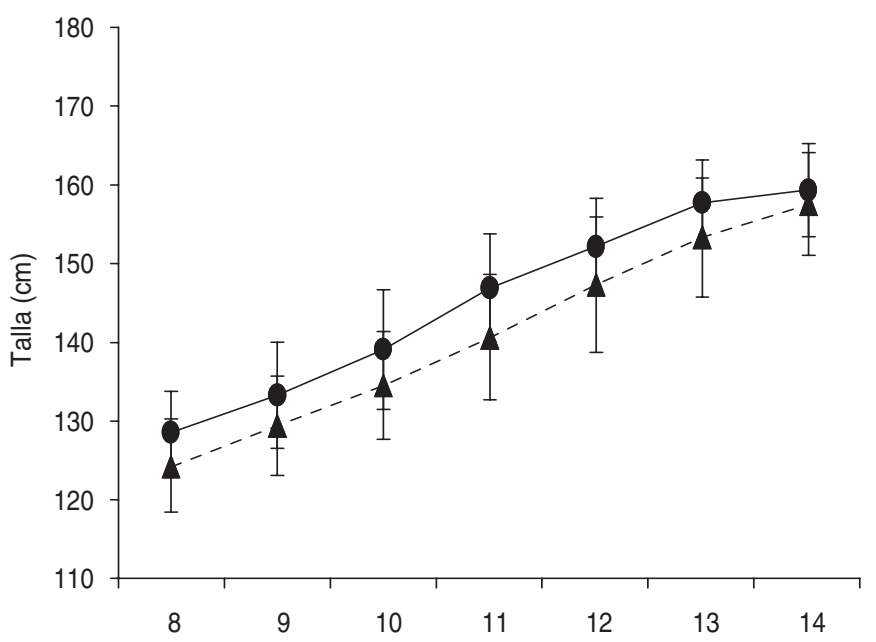

Edad, años 
realizados sobre tendencia secular han evidenciado un cambio positivo en la talla, con un aumento medio de $1,5 \mathrm{~cm}$ por década (26-28). Coincidentemente, en el presente estudio se observó un incremento medio en la talla de $3 \mathrm{~cm}$ en los varones y $4 \mathrm{~cm}$ en las mujeres, con respecto a los valores de referencia nacionales. Estas diferencias llegaron a ser más pronunciadas en los varones, con valores máximos de 5,6 y $7,7 \mathrm{~cm}$ a los 13 y 14 años, respectivamente. En las mujeres la tendencia fue similar, con un valor máximo de $6,4 \mathrm{~cm}$ a $\operatorname{los} 11$ años de edad (figura 2).
Para los países en desarrollo, la Organización Mundial de la Salud (OMS) recomienda emplear la talla para la edad como un indicador que permite planificar un amplio espectro de intervenciones, tanto sanitarias como económicas (29). En nuestro estudio, las diferencias entre las prevalencias de retraso del crecimiento calculadas según ambas referencias alcanzaron un $16 \%$, lo que refleja cuadros disímiles del estado nutricional de la población evaluada. Estos hallazgos pueden repercutir tanto en la evaluación del crecimiento y el estado nutricional como en la identificación de las poblaciones objeto de programas de intervención. Asimismo, apuntan a la necesidad de construir una nueva referencia nacional que sea representativa de las distintas regiones del país.

Agradecimientos. Este trabajo fue parcialmente financiado con fondos provenientes de la Universidad Nacional de La Plata (UNLP). Los autores agradecen la colaboración de Adelaida Kraan, Adelaida Colángelo, Carlos Golijow, Luis Pérez y Sebastián Picco.

\section{REFERENCIAS}

1. Edwards J, Morse JM. Assessment of growth: is one international growth reference sufficient? Public Health Nutr. 1989;6:35-42.

2. Waterlow JC, Bucina R, Keller W, Lane JM, Nichaman MZ, Tanner JM. The presentation and use of height and weight data for comparing the nutritional status of groups of children under the age of 10 years. Bull WHO. 1977;55:489-98.

3. Van Loon H, Saverys V, Vuylsteke JP, Vlietinck RF, Eeckels R. Local versus universal growth standards: the effect of using NCHS as universal reference. Ann Hum Biol. 1986; 13:347-57.

4. Seth V, Rai A, Gupta M, Semwal OP, Patnaik KK, Sundaram KR. Construction of growth reference standards for urban slum children in developing countries. Indian Pediatr. 1990; 27:1081-7.

5. Ayatollahi SM, Ahmadi K. Infants' growth charts for southern Iran. Am Hum Biol. 2001; 28:337-45.

6. Fredriks AM, van Buuren S, Jeurissen SE, Dekker FW, Verloove-Vanhorick SP, Wit JM. Height, weight, body mass index and pubertal development references for children of Moroccan origin in The Netherlands. Acta Paediatr. 2004;93:817-24.

7. Jhonston FE, Ouyang Z. Choosing appropriate reference data for the anthropometric assessment of nutritional status. En: Himes $\mathrm{JH}$, ed. Anthropometric assessment of nutritional status. New York: Willey Liss; 1991. Pp. 337-46.

8. De Onis M, Yip R. The WHO Growth Chart: Historical Considerations and Current Scientific Issues. En: Porrini M, Walter P, eds. Nutrition in pregnancy and growth. Bibl Nutr Dieta. Bassel: Karger; 1996. No 53, Pp. 74-89.
9. Roche AF. Secular trends in human growth maturation, and development. Soc Res Child Dev. 1979;44(3-4), Series 179.

10. Sociedad Argentina de Pediatría. Guías para la evaluación del crecimiento. 2. ${ }^{a}$ ed. Buenos Aires: Sociedad Argentina de Pediatría; 2001.

11. Cusminsky M, Castro E, Azcona LCh, Jubany E, Mele E. Estudio longitudinal del crecimiento y desarrollo del niño en La Plata. Comisión de Investigaciones Científicas de la Provincia de Buenos Aires; 1966.

12. Cusminsky M, Castro E, Lozano G, Lejarraga $\mathrm{H}$, Spotti M, Porfiri N, et al. Investigación del crecimiento y desarrollo del niño de 4 a 12 años. Buenos Aires: Ministerio de Bienestar Social, Comisión de Investigaciones Científicas; 1974.

13. Funes Lastra P, Agrelo F, Guita S, Chiquilito FC de, Borjadello LT de, Videla N, et al. Desarrollo de niños normales de la ciudad de Córdoba a través de una muestra representativa. Córdoba, AR: Universidad Nacional de Córdoba; 1975.

14. Lejarraga H, Orfila G. Estándares de peso y estatura para niñas y niños argentinos desde el nacimiento hasta la madurez. Arch Argent Pediatr. 1987;85:209-22.

15. Del Pino M, de Olivera N, Lejarraga H. Vigencia de los estándares nacionales de peso y estatura de 0 a 5 años. Arch Argent Pediatr. 2003;101:351-6.

16. Lohman TG, Roche AF, Martorell R. Anthropometric standardization reference manual. Champaign, IL: Human Kinetics Books; 1988.

17. Prieto L, Lamarca R, Casado A. La evaluación de la fiabilidad en las observaciones clínicas: el coeficiente de correlación intraclase. Med Clin (Barcelona). 1998;110:142-5.
18. Frisancho AR. Anthropometric standards for the assessment of growth and nutritional status. Ann Arbor: University of Michigan Press; 1990.

19. Gorstein J, Sullivan K, Yip R, de Onis M, Trowbridge F, Fajans P, et al. Issues in the assessment of nutritional status using anthropometry. Bull World Health Organ. 1994;72: 273-83.

20. Clark ML. Los valores $P$ y los intervalos de confianza: ¿en qué confiar? Rev Panam Salud Publica. 2004;15:293-6.

21. De Onis M, Monteiro C, Akré J, Clugston G. The world wide magnitude of protein-energy malnutrition: an overview from the WHO Global Data Base on Child Growth. Bull World Health Organ. 1993;71:703-12.

22. Eckhardt CL, Adair LS. Differences in stunting prevalences calculated from two similar growth references may be large and inconsistent in undernourished children. Ann Hum Biol. 2002;29:566-78

23. Castilho LV, Larh MM. Secular trends in growth among urban Brazilian children of European descent. Am Hum Biol. 2001;28: 564-74.

24. Barbieri Marmo D, Porto Zambon M, Moreno Morcillo A, Guimarey LM. Téndencia secular de crescimento em escolares de Paulina, São Paulo-Brasil (1979/80-1993/94). Rev Assoc Med Bras. 2004;50:386-90.

25. Stein AD, Barnhart HX, Wang M, Hoshen MB, Ologoudou K, Ramakrishnan U, et al. Comparison of linear growth patterns in the first three years of life across two generations in Guatemala. Pediatrics. 2004;113:270-5.

26. Lejarraga H. Peso y talla de 15.214 adolescentes de todo el país. Tendencia secular. Arch Argent Pediatr. 1986;64:219-35. 
27. Lejarraga H, Abeyá Gilardón EO, Andrade $\mathrm{JH}$, Boggero H. Evaluación del peso y la talla en 88.861 varones de 18 años de la República Argentina (1987). Arch Argent Pediatr. 1991;89:185-92.

28. Andrade JH, Calvo EB, Longo EN, Marconi EN. Evaluación del estado nutricional de la población de varones de 18 años. Argentina,
1992-1993. En: Calvo EB, ed. Estudios antropométricos en la población infanto-juvenil. República Argentina, 1993-1996. Buenos Aires: Ministerio de Salud y Acción Social de la Nación; 1999.

29. World Health Organization. Physical status: the use and interpretation of anthropometry. Report of a WHO Expert Committee. Geneva:
World Health Organization; 1995. (Technical Report Series, No. 854).

Manuscrito recibido el 6 de octubre de 2004. Aceptado para publicación, tras revisión, el 25 de mayo de 2005

ABSTRACT Objective. To evaluate if using an international growth reference versus a national growth reference leads to differences in the prevalences of low weight-for-height (wasting) and low height-for-age (stunting) in a sample of children 8 to 14 years old.

Differences in the prevalence of malnutrition resulting from using a national growth reference or an international growth reference
Methods. A cross-sectional study was carried out with a sample of 1470 schoolchildren (both boys and girls) in the city of La Plata, in the province of Buenos Aires, Argentina. Based on the data on weight and height, $Z$-scores were calculated with regard to the reference values from the United States of America's first and second National Health and Nutrition Examination Survey (NHANES I and NHANES II), and the reference values for Argentina from the Argentine Pediatric Society. The prevalences of wasting and stunting were calculated, using as the cutoff point two standard deviations below the median of the reference. The prevalences were compared using the chi-squared test with Yates' correction, and odds ratios (ORs) and 95\% confidence intervals (95\% CIs) were calculated.

Results. The international reference was positively associated with a higher prevalence of stunting in the entire sample (OR $=5.12 ; 95 \% \mathrm{CI}: 3.27$ to 8.05), in the boys $(\mathrm{OR}=4.36 ; 95 \% \mathrm{CI}: 2.43$ to 7.93$)$, and in the girls (OR $=6.32 ; 95 \% \mathrm{CI}: 3.10$ to 13.27$)$. The prevalence of wasting was similar with both references. The distribution of heightfor-age was skewed to the left with respect to the international reference and to the right with respect to the Argentine reference, while the distribution of weight-forheight overlapped both references.

Conclusions. The differences in the prevalences of stunting calculated according to the two references that were used indicate a different nutritional status for the population studied. The increase in the height of the children with respect to the Argentine reference points to the presence of a positive secular trend, and the increase can explain part of the differences in the prevalence of stunting.

Key words Reference standards, nutritional status, malnutrition. 